\title{
Running impact forces: from half a leg to holistic understanding - comment on Nigg et al.
}

\author{
Kenneth P. Clark', Andrew B. Udofa², Laurence J. Ryan² \& Peter G. Weyand ${ }^{2, *}$ \\ 1 Department of Kinesiology, West Chester University, West Chester PA, USA \\ 2 Locomotor Performance Laboratory, Department of Applied Physiology and Wellness, Southern Methodist University, Dallas, Texas, USA \\ * Corresponding author: Department of Applied Physiology and Wellness, Southern Methodist University, Dallas, Texas 75206, USA \\ Email: pweyand@smu.edu
}

\section{COMMENTARY}

\section{Article History:}

Submitted $7^{\text {th }}$ February 2018

Accepted $1^{\text {st }}$ March 2018

Published $18^{\text {th }}$ April 2018

Handling Editor:

Markus Tilp

Karl-Franzens-University Graz, Austria

Editor-in-Chief:

Martin Kopp

University of Innsbruck, Austria

\begin{abstract}
Running impact forces have immediate relevance for the muscle tuning paradigm proposed here and broader relevance for overuse injuries, shoe design and running performance. Here, we consider their mechanical basis. Several studies demonstrate that the vertical ground reaction force-time (vGRFT) impulse, from touchdown to toe-off, corresponds to the instantaneous accelerations of the body's entire mass $(\mathrm{Mb})$ divided into two or more portions. The simplest, a two-mass partitioning of the body (lower-limb, $M_{1}=0.08 \cdot M_{b}$; remaining mass, $M_{2}=0.92 \cdot M_{b}$ ) can account for the full vGRFT waveform under virtually all constant-speed, level-running conditions. Model validation data indicate that: 1) the non-contacting mass, $\mathrm{M}_{2^{\prime}}$ often accounts for one-third or more of the early "impact" portion of the vGRFT, and 2) extracting a valid impact impulse from measured force waveforms requires only lower-limb motion data and the fixed body mass fraction of 0.08 for $M_{1}$.
\end{abstract}

Keywords:

Two-mass model - effective mass - ground reaction forces - running performance - spring-mass model

Citation:

Clark , K. P. , Udofa A. B. , Ryan, L. J. \& Weyand , P. G. (2018): Running impact forces: from half a leg to holistic understanding - comment on Nigg et al. Current Issues in Sport Science, 3:107. doi: 10.15203/CISS_2018.107

This is a commentary on a CISS report article authored by Nigg, B. M., Mohr, M. \& Nigg, S. R. (2017). Muscle tuning and preferred movement path - a paradigm shift. Current Issues in Sport Science, 2:007. doi: 10.15203/CISS_2017.007

\section{Impact Forces Revisited}

Dr. Nigg and colleagues deserve commendation for efforts that have endowed the area of running biomechanics with a sizeable body of empirical observations. These observations have, and undoubtedly will, continue to inform work on a broad range of topics that include running injuries, running shoes and the relationship between the two. Their willingness to confront the experimental challenges involved in studying a largely unpredictable phenomenon like running injuries deserves particular praise.

Here, we focus on the impact force conclusions offered by Nigg, Mohr and Nigg (2017) in their target article. While their contribution purports a lack of importance in overuse injury etiology, there are compelling scientific reasons to consider their basis and importance from an independent, contemporary perspective. These are: 1 ) the existence of credible evidence supporting a running impact force-overuse injury link (Daoud, Geissler, Wang, Saretsky, Daoud, \& Liebermann, 2012; Milner, Ferber, Pollard, Hamill, \& Davis, 2006), 2) the direct effect of impact forces on bodily motion and performance (Clark \& Weyand, 2014), and 3) the need for valid quantification to advance general understanding and inform specific applications. One noteworthy application is the input signal required by the muscle-tuning paradigm Nigg et al. advance in their target article here. More broadly, the inability to quantify running impact forces recently noted by Nigg and colleagues elsewhere (Baltich, Maurer \& Nigg, 2015) is obviously a direct impediment to reaching firm conclusions regarding their importance. 
From our perspective, the development of a quantitative understanding has been at least partially impeded by two assumptions that have framed the study of running impact forces for decades. These are: 1) assuming that the early portion of the vertical ground reaction force-time waveform can be attributed to a small fraction (i.e. an "effective mass") of the body's total mass $\left(M_{b}\right)$ while ignoring the rest (Chi \& Schmitt, 2005; Denoth, 1986; Derrick, 2004; Lieberman, Venkadesan, Werbel, Daoud, D'Andrea, Davis, Mang'eni \& Pitsilades, 2010 ; Nigg, 2010; Nigg, Mohr, Nigg, 2017), and 2) assuming that the impact impulse can be quantified using the localized force peak often visible on the rising edge of the measured waveform (Figure 1). Three studies indicate that these assumptions obscure the mechanics of the impact event.

The first study was provided by Bobbert, Schamhardt and Nigg (1991) more than 25 years ago. These investigators demonstrated that the instantaneous accelerations of seven body mass components (comprising $100 \%$ of $\mathrm{M}_{\mathrm{b}}$ : right and left foot, shank, and upper leg components plus a combined head-arms-torso mass) acquired from motion data, can be summed to provide a close match to the measured total vertical ground reaction force-time (VGRFT) waveform during slow and moderate speed running (i.e. $F_{z 1}+F_{z 2}+F_{z 3}+F_{z 4}+F_{z 5+} F_{z 6}+F_{z 7}=F_{z \text {-totali }}$ where $z$ designates the vertical component of the ground reaction force). This noteworthy experimental accomplishment was based on Newton's $2^{\text {nd }}$ Law including the first-principle recognition that the measured vGRFT waveform must somehow correspond to the instantaneous accelerations of $100 \%$ of the body's mass. The second insightful study was the detailed temporal and spatial analysis of the rising edge of the VGRFT undertaken by Shorten and Mientjes (2011). From pressure mapping data on the sole of the foot and frequency analyses of measured waveforms, these investigators also concluded that the body's entire mass contributed to the rising edge of the waveform. Per their title, they concluded that the localized, rising-edge waveform force peak widely attributed to heel impact, is in fact, "neither heel, nor impact" during heel-toe running.

The most recent of the three studies involved an experimental effort from our laboratory (Clark, Ryan \& Weyand, 2017) that, like Bobbert et al. adopted a Newtonian approach. We did so with the goal of identifying the simplest partitioning of the body that might account for the vGRFT waveform in full. Our efforts led to the two body-mass component, two-impulse waveform explanation illustrated in Figure 1. Ultimately, this approach was able to predict 500 measured vGRFT waveforms acquired at speeds from 3.0 to $11.0 \mathrm{~m} / \mathrm{s}$ regardless of the runner's foot-strike mechanics. Due to the model's conciseness, only three inputs are required to generate the waveforms from a runner's gait mechanics: contact time, aerial time, and the vertical acceleration of the lower limb. The close agreement between model-generated and measured VGRFT waveforms $\left(R^{2}=0.95\right)$ supports the general validity of the two-mass, twoimpulse explanation for their mechanical basis.

\section{The Rising Edge of the vGRFT Waveform: Impact Is Not Enough}

Clearly, additional experimental work remains to test and refine the existing Newtonian explanations for running vGRFT waveforms. However, the two studies that have successfully linked bodily motion to running ground reaction forces share the foundational recognition that the waveform represents the summed acceleration of $100 \%$ of body mass.

The holistic Newtonian view that emerges for the rising-edge of the total vGRFT waveform, broadly conceived elsewhere as an "impact-only" event, is illustrated in Figure 1. The data appearing in the figure have been adapted from original vGRFT data acquired at a speed of 5.0 meters per second from a runner with heel-strike mechanics. As illustrated, the body's full mass contributes to the rising edge of the waveform. Accurately predicting the magnitude and timing of the localized peak, for example, requires summing the impulse contributions of the model's body mass components. Per the illustration, correct prediction of the overall impact mechanics using the two masses in our model relies heavily on the kinematic data used to determine $\Delta \mathrm{t}$ from the period elapsing between the instant of initial foot-ground contact and subsequent time at which mass $M_{1}$ slows to a vertical velocity of zero. Correct identification of the localized rising-edge peaks for heel-strikers at all speeds and competitive sprinters at faster ones as previously reported would have been virtually impossible (Clark, Ryan \& Weyand, 2017, Figures 5, 6 and 7) without both: 1) accurate

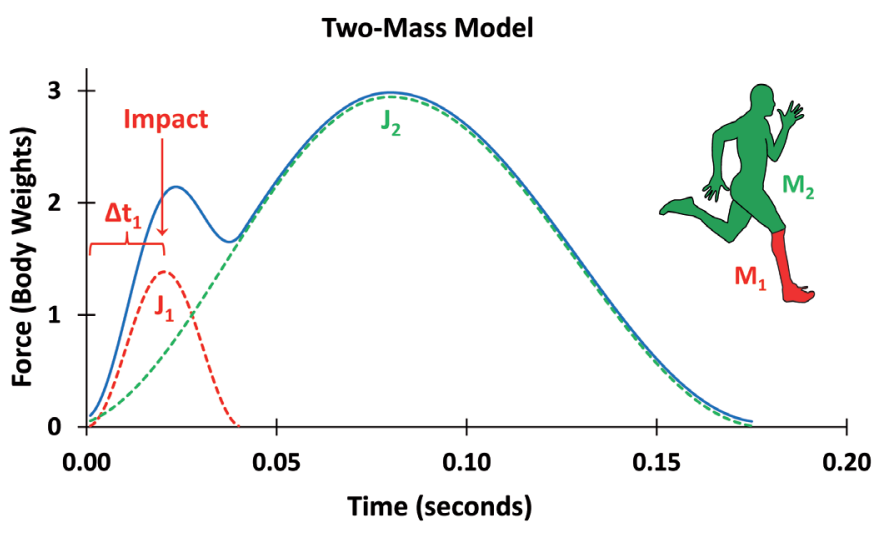

Figure 1: Running vertical ground reaction forces can be quantified as the sum of two impulses: a relatively brief impact impulse $\left(\mathrm{J}_{1}\right)$ that corresponds to the deceleration of the foot and shank $\left(M_{1}=0.08 \cdot M_{b}\right)$, and a larger, longer-duration impulse $\left(\mathrm{J}_{2}\right)$ that corresponds to the acceleration of the remainder of the body's mass $\left(\mathrm{M}_{2}=0.92 \cdot \mathrm{M}_{\mathrm{b}}\right)$ throughout the stance period. Both impulses begin at the instant of touchdown and contribute substantially to the rising-edge of the force-time waveform. Note that impact impulse, $J_{1}$, is quantified from $8 \%$ of $\mathrm{M}_{\mathrm{b}}$ and the time $\Delta \mathrm{t}_{1}$ at which the ankle reaches its minimum vertical position. 
kinematic data, and 2) a model capable of predicting the significant variability in the timing and magnitude of rising-edge force peaks across scores of different footfalls.

Figure 1 also reveals the specific manner in which the assumptions required by the effective mass techniques obscure the mechanical basis of the rising edge of the waveform. In the footfall illustrated, the effective mass approach assumes that mass $M_{2}$ would make little or no contribution to the risingedge impulse up to the localized force peak. However, as illustrated, $M_{2}$ is actually responsible for roughly one-third of the total impulse over this early period of this illustrated waveform. During slow and moderate speed fore-foot strikes, the impulse contributions of mass $M_{2}$ actually exceed those of $M_{1}$, primarily because the impact period $\Delta \mathrm{t}$, is relatively longer (Clark, Ryan \& Weyand, 2017, Figures 5 and 6, Table 2). Also evident in the figure is that the localized peak on the total VGRFT waveform is not simultaneous with the peak of impact impulse $J_{1}$ as implicitly assumed by effective mass quantification techniques. Rather, the time-dependent contributions of impulse $\mathrm{J}_{2}$ cause the total waveform peak to occur at a later point in time than the $J_{1}$ impulse peak. In the case of most forefoot strike waveforms, the longer $\Delta t_{1}$ period results in the rising edge of the measured waveform lacking a localized force peak altogether (Clark, Ryan \& Weyand, 2014; Clark, Ryan \& Weyand, 2017, Figure 6, Table 2).

\section{Impact Forces and New Paradigms: Retro- and Prospective Considerations}

Intuitive appeal and computational simplicity may be responsible for the common conceptualization and quantification of the rising edge of human running vGRFT waveforms as an impact-only phenomenon. However, the works synthesized here: 1) provide a valid mechanical basis for the vGRFTs waveforms based on the body's entire mass, and 2) offer quantitative methods that apply over essentially all level-speed and footstrike conditions. A two-mass partitioning of the human body allows the full running vGRFT waveform to be predicted from gait motion. The two-mass approach also allows the impact portion of the impulse to be extracted from measured vGRFT waveforms. Doing so requires only motion data from the ankle and the fixed lower-limb mass fraction identified for $M_{1}$.

Finally, we applaud Nigg, Mohr and Nigg for proposing muscle tuning and movement path paradigms in an effort to advance basic and applied understanding of running mechanics. We share their view that evaluating these paradigms will be a major and lengthy experimental undertaking. One useful tool for these efforts, directly in the case of muscle tuning and indirectly for preferred movement paths, is the holistic quantitative understanding of impact forces that is currently available.

\section{Funding}

This work was supported in part by a US Army Medical and Materiel Command award [W81XWH-12-2-0013] to PGW.

\section{Competing Interests}

The authors declare competing financial interests. Peter Weyand, Laurence Ryan and Kenneth Clark are the inventors of US Patent \#8363891 which is owned by SMU and contains scientific content related to that presented in the manuscript. The patent is licensed to SoleForce LLC in which the three aforementioned individuals are equity partners.

\section{Data Availability Statement}

All relevant data are within the paper.

\section{References}

Baltich J., Maurer C., Nigg B.M. (2015). Increased vertical impact forces and altered running mechanics with softer midsole shoes. PLOS One, doi:10.1371/journal. pone.012519610.15203/CISS_2016.101

Bobbert, M.F., Schamhardt, H.C., and Nigg, B.M. (1991). Calculation of vertical ground reaction force estimates during running from positional data. Journal of Biomechanics. 24, 1095-1105.

Chi, K.J., and Schmitt, D. (2005). Mechanical energy and effective foot mass during impact loading of walking and running. Journal of Biomechanics. 38, 1387-1395.

Clark, K.P., Weyand, P.G. (2014). Are running speeds maximized with simple-spring stance mechanics? Journal of Applied Physiology. 117, 604-615.

Clark, K.P., Ryan, L.J., Weyand, P.G. (2014). Foot speed, foot-strike and footwear: linking gait mechanics and running ground reaction forces. Journal of Experimental Biology. 217, 20372040.

Clark, K.P., Ryan, L.J., Weyand, P.G. (2017). A general relationship links gait mechanics and running ground reaction forces. Journal of Experimental Biology. 220(2), 247-258.

Daoud A.I., Geissler G.J., Wang F., Saretsky J., Daoud Y.A., Lieberman D.E. (2012) Foot Strike and Injury Rates in Endurance Runners: a retrospective study. Medicine and Science in Sports and Exercise. 44(7), 1325-1334.

Denoth, J. (1986). Load on the locomotor system and modeling. In Biomechanics of Running Shoes (ed. B. M. Nigg), pp. 63116. Illinois: Human Kinetics Publishers, Inc.

Derrick, T. R. (2004). The effects of knee contact angle on impact forces and accelerations. Medicine and Science in Sports and Exercise. 36, 832-837. 
Lieberman, D.E., Venkadesan, M., Werbel, W.A., Daoud, A.I., D'Andrea, S., Davis, I.S., Mang'eni, R.O., and Pitsiladis, Y. (2010). Foot strike patterns and collision forces in habitually barefoot versus shod runners. Nature 463, 531-535.

Milner C.E., Ferber R., Pollard C.D., Hamill J., Davis I.S. (2006). Biomechanical factors associated with tibial stress fractures in female runners. Medicine and Science in Sports and Exercise, pp. 38(2), 323-328.

Nigg, B.M. (2010). Biomechanics of Sport Shoes. Calgary, AB: Topline Printing, Calgary. p. 1-41.

Nigg B.M., Mohr M.M., Nigg S. (2017) Muscle tuning and preferred movement path: a paradigm shift. Current Issues in Sport Science. DOI 10.15203/CISS_2017.007

Shorten, M., and Mientjes, M.I.V. (2011). The "heel impact" force peak during running is neither "heel" nor "impact" and does not quantify shoe cushioning effects, Footwear Science. 3, 41-58. 